\title{
On the dimension of Voisin sets in the moduli space of abelian varieties
}

\author{
E. Colombo ${ }^{1}$ • J. C. Naranjo² . G. P. Pirola ${ }^{3}$
}

Received: 20 April 2020 / Revised: 7 November 2020 / Accepted: 19 December 2020 /

Published online: 12 January 2021

(c) The Author(s) 2021

\begin{abstract}
We study the subsets $V_{k}(A)$ of a complex abelian variety $A$ consisting in the collection of points $x \in A$ such that the zero-cycle $\{x\}-\left\{0_{A}\right\}$ is $k$-nilpotent with respect to the Pontryagin product in the Chow group. These sets were introduced recently by Voisin and she showed that $\operatorname{dim} V_{k}(A) \leq k-1$ and $\operatorname{dim} V_{k}(A)$ is countable for a very general abelian variety of dimension at least $2 k-1$. We study in particular the locus $\mathcal{V}_{g, 2}$ in the moduli space of abelian varieties of dimension $g$ with a fixed polarization, where $V_{2}(A)$ is positive dimensional. We prove that an irreducible subvariety $\mathcal{Y} \subset \mathcal{V}_{g, 2}$, $g \geq 3$, such that for a very general $y \in \mathcal{Y}$ there is a curve in $V_{2}\left(A_{y}\right)$ generating $A$ satisfies $\operatorname{dim} \mathcal{Y} \leq 2 g-1$. The hyperelliptic locus shows that this bound is sharp.
\end{abstract}

Mathematics Subject Classification $14 \mathrm{~K} 10 \cdot 14 \mathrm{C} 15$

Dedicated to the memory of our friend Alberto Collino.

Communicated by Vasudevan Srinivas.

E. Colombo and G.P. Pirola are members of Gnsaga (INDAM) and are partially supported by PRIN projectModuli spaces and Lie theory (2017), G.P. Pirola is partially supported by MIUR: Dipartimenti di Eccellenza Program (2018-2022) - Dept. of Math. Univ. of Pavia. Naranjo was partially supported by the Proyecto de Investigación MTM2015-65361-P and PID2019-104047GB-100.

$凶$ J. C. Naranjo

jcnaranjo@ub.edu

E. Colombo

elisabetta.colombo@unimi.it

G. P. Pirola

gianpietro.pirola@unipv.it

1 Dipartimento di Matematica, Università degli Studi di Pavia, Via Cesare Saldini 50, 20133 Milan, Italy

2 Departament de Matemàtiques i Informàtica, Universitat de Barcelona, Gran Via 585, 08007 Barcelona, Spain

3 Dipartimento di Matematica, Università degli Studi di Pavia, Via Ferrata 1, 27100 Pavia, Italy 


\section{Introduction}

Claire Voisin in [12] defines the subset $V_{k}(A)$ of a complex abelian variety $A$ consisting in the collection of points $x \in A$ such that the zero-cycle $\{x\}-\left\{0_{A}\right\}$ is $k$-nilpotent with respect to the Pontryagin product in the Chow group:

$$
V_{k}(A):=\left\{x \in A \mid\left(\{x\}-\left\{0_{A}\right\}\right)^{* k}=0 \text { in } C H_{0}(A)_{\mathbb{Q}}\right\} .
$$

Here we have denoted by $\{x\}$ the zero-cycle of degree 1 corresponding to the point $x \in A$. These are naturally defined sets in the sense that they exist in all the abelian varieties, are functorial and move in families. Moreover they are related with the gonality of the abelian variety itself (the minimal gonality of a curve contained in $A$ ) in a natural way.

We consider the following subsets of the moduli space of abelian varieties of dimension $g$ with a polarization of type $\delta$ :

$$
\mathcal{V}_{g, k, l}=\left\{A \in \mathcal{A}_{g}^{\delta} \mid \operatorname{dim} V_{k}(A) \geq l\right\}
$$

Since the sets $V_{k}$ are naturally defined, then $\mathcal{V}_{g, k, l}$ is a union of countably many closed subvarieties of $\mathcal{A}_{g}^{\delta}$. Hence it makes sense to ask about its dimension. Put $\mathcal{V}_{g, k}:=\mathcal{V}_{g, k, 1}$. For an abelian subvariety $B \subset A$ the inclusion $V_{k}(B) \subset V_{k}(A)$ holds and a wellknown theorem of Bloch implies that $B=V_{k}(B)$ if $\operatorname{dim} B+1 \leq k$, hence in this situation $B \subset V_{k}(A)$. These are in some sense "degenerated examples". In this paper we concentrate in the case $k=2$ and we take care of the non-degenerate case, that is, we will assume that $V_{2}(A)$ contains some curve generating the abelian variety $A$.

Our main result is:

Theorem 1.1 Let $g \geq 3$ and consider an irreducible subvariety $\mathcal{Y} \subset \mathcal{V}_{g, 2}$ such that for a very general $y \in \mathcal{Y}$ there is a curve in $V_{2}\left(A_{y}\right)$ generating $A_{y}$. Then $\operatorname{dim} \mathcal{Y} \leq 2 g-1$.

This result is sharp due to the fact that the hyperelliptic locus is contained in $\mathcal{V}_{g, 2}$, see Sect. 2. In fact, the motivation for this study is to understand the geometrical meaning of the positive dimensional components in $V_{2}$. Our result gives some evidence that there is a link between the existence of hyperelliptic curves in abelian varieties and the fact that $V_{2}$ is positive dimensional. We remark that the statement of the theorem (1.1) was suggested by the analogous result in [7] concerning hyperelliptic curves.

Section 2 is devoted to give some preliminaries and some useful properties of the loci $V_{k}(A)$ focusing specially on the case $k=2$. A remarkable property is that $V_{2}(A)$ is the preimage of the orbit of the image of the origin with respect to rational equivalence in the Kummer variety $\operatorname{Kum}(A)$. We also prove the following interesting facts (see Corollaries 2.12 and 2.13)

Proposition 1.2 For any abelian variety A the inclusion

$$
V_{k}(A)+V_{l}(A) \subset V_{k+l-1}(A)
$$


holds for all $1 \leq k, l \leq g$. Moreover if $C$ is a hyperelliptic curve of genus $g$, and $J(C)$ be its Jacobian variety, then for all $1 \leq k \leq g+1$, we have that $\operatorname{dim} V_{k}(J C)=k-1$ (the maximal possible value).

The rest of the paper is devoted to the proof of the main theorem. The beginning follows closely the same strategy as in [7] since we reduce to prove the vanishing of a certain adjoint form. The novelty here is that we prove this vanishing by using the action of a family of rationally trivial zero-cycles as in the spirit of Mumford and Roitman results revisited by Bloch-Srinivas, Voisin and others.

More precisely, we can assume that there is relative map $f: \mathcal{C} \longrightarrow \mathcal{A}$ of curves in abelian varieties over a base $\mathcal{U}$ such that $f\left(C_{y}\right) \subset V_{2}\left(A_{y}\right)$ generates the abelian variety $A_{y}$ for all $y \in \mathcal{U}$. We use the properties of the sets $V_{2}\left(A_{y}\right)$ to construct a cycle on the family of abelian varieties which acts trivially on the differential forms. Then, by using deformation of differential forms as in [7] we compute the so-called adjunction form in a generic point of the family. This technique can be traced-back to [5], where this procedure is introduced for the first time. In Sect. 4 we assume that, by contradiction, the dimension of the family is $\geq 2 g$ and we prove that this implies the existence of a non-trivial adjoint form. This contradicts the results on Sect. 3 .

\section{Preliminaires on the subsets $V_{k}$ of an abelian variety $A$}

\subsection{On the dimension of $V_{k}$}

The most part of this subsection comes from [12], where the sets $V_{k}(A)$ appear for the first time.

Definition 2.1 Let $A$ be an abelian variety and denote by $\mathrm{CH}_{0}(A)$ the Chow group of zero-cycles in $A$ with rational coefficients. We also denote by $*$ the Pontryagin product in the Chow group. Given a point $x \in A$, we put $\{x\}$ for the class of $x$ in $C H_{0}(A)$. Then we define the Voisin sets (cf. [12]):

$$
V_{k}=V_{k}(A):=\left\{x \in A \mid\left(\{x\}-\left\{0_{A}\right\}\right)^{* k}=0\right\}
$$

It is known that the set $V_{k}$ is a countable union of closed subvarieties of $A$. A typical way to obtain points in $V_{k}$ is given by the following Proposition:

Proposition 2.2 ([12, Prop. (1.9)]) Assume that $\left\{x_{1}\right\}+\cdots+\left\{x_{k}\right\}=k\left\{0_{A}\right\}$ in $C H_{0}(A)$ for some points $x_{i} \in A$, then for all $i$ we have $x_{i} \in V_{k}$.

Since the orbits w.r.t. rational equivalence $\left|k\left\{0_{A}\right\}\right|$ are hard to compute there are only a few examples of positive dimensional components in $V_{k}(A)$ that we can construct from this proposition. The simplest instance of this comes from a $k$-gonal curve $C$ contained in $A$ such that there is a totally ramified point $p$ for the degree $k$ map $f: C \longrightarrow \mathbb{P}^{1}$. Translating we can assume that $p$ is the origin $0_{A}$ and then the fibers of $f$ provide a 1-dimensional component in the symmetric product, $k$ times, of $A$. Therefore, by the proposition above, we obtain that $C$ is contained in $V_{k}(A)$. 
Observe that the sets $V_{k}$ are invariant under isogenies, hence these positive dimensional components also appear in many other abelian varieties. In particular for any integer $n$, we have that $n_{*} V_{2}(A) \subset V_{2}(A)$.

Remark 2.3 We have the following properties:

(a) All the abelian varieties containing hyperelliptic curves have positive dimensional components in $V_{2}$.

(b) A very well known theorem of Bloch (see [3]) implies that

$$
V_{g+1}(A)=A
$$

hence the natural filtration

$$
V_{1}(A) \subset V_{2}(A) \subset \cdots \subset V_{g}(A) \subset V_{g+1}(A)=A
$$

has at most $g$ steps. It is natural to ask what is the behaviour of the dimension of $V_{k}(A)$, with $k \leq g$, for very general abelian varieties, and which geometric properties of $A$ codify these sets.

(c) Assume that $B \subset A$ is an abelian subvariety, then $V_{k}(B) \subset V_{k}(A)$. In particular, if $k \geq \operatorname{dim} B+1$, then $B \subset V_{k}(A)$. For instance: all the elliptic curves in $A$ passing through the origin are contained in $V_{2}(A)$.

(d) Let $C$ be a smooth quartic plane curve and let $p$ be a flex point with tangent $t$, then $t \cdot C=3 p+q$ and the projection from $q$ provides a collection of zero-cycles of degree 3 in $J C$ rationally equivalent to $3\left\{0_{J C}\right\}$, then $C \subset V_{3}(J C)$. Using isogenies we get that there are in fact a countably number of curves in $V_{3}(A)$ for a very general abelian variety of dimension 3 .

The following is proved in Theorem (0.8) of [12] by using some ideas from [6] and improving the techniques of [8]:

Theorem 2.4 Let $A$ be an abelian variety of dimension $g$. Then:

(a) $\operatorname{dim} V_{k}(A) \leq k-1$.

(b) If $A$ is very general and $g \geq 2 k-1$ we have that $\operatorname{dim} V_{k}(A)=0$.

In the specific case of $V_{2}(A)$ we have the following properties.

Proposition 2.5 Let $A$ be an abelian variety and let $K u m(A)$ be its Kummer variety.

(a) We have the equality $V_{2}(A)=\left\{x \in A \mid\{x\}+\{-x\}=2\left\{0_{A}\right\}\right\}$.

(b) Let $\alpha: A \longrightarrow K u m(A)$ be the quotient map. Then

$$
V_{2}(A)=\alpha^{-1}\left(\left\{y \in \operatorname{Kum}(A) \mid\{y\} \sim_{\text {rat }}\left\{\alpha\left(0_{A}\right)\right\}\right\}\right)
$$

Proof Part (a) follows from the observation that

$$
\left(\{x\}-\left\{0_{A}\right\}\right)^{* 2}=\{2 x\}-2\{x\}+\left\{0_{A}\right\}=0
$$

is equivalent, translating with $-x$, to $\{x\}+\{-x\}=2\left\{0_{A}\right\}$. 
To prove (b) we first see that $x \in V_{2}(A)$ if and only if $\{\alpha(x)\} \sim_{\text {rat }}\left\{\alpha\left(0_{A}\right)\right\}$. Indeed, assume that $\{x\}+\{-x\}=2\left\{0_{A}\right\}$, applying $\alpha$ we get that $2\{\alpha(x)\} \sim_{\text {rat }} 2\left\{\alpha\left(0_{A}\right)\right\}$. Since $\operatorname{Alb}(\operatorname{Kum}(A))=0$ the Chow group has no torsion (see [9]) therefore $\{\alpha(x)\} \sim_{\text {rat }}$ $\left\{\alpha\left(0_{A}\right)\right\}$. In the opposite direction, if $\{\alpha(x)\} \sim_{\text {rat }}\left\{\alpha\left(0_{A}\right)\right\}$ we apply $\alpha^{*}$ at the level of Chow groups and we obtain that $x \in V_{2}(A)$. Hence $V_{2}(A)$ is the pre-image by $\alpha$ of the points rationally equivalent to $\alpha\left(0_{A}\right)$.

\subsection{Relation with the Chow ring}

In this part we collect some computations on 0 -cycles on abelian varieties which are more or less implicit in $[1,2,12]$.

Let us recall first some facts on the Chow group (with rational coefficients) of an abelian variety $A$ of dimension $g$ which are proved in [2]. Let us define the subgroups:

$$
C H_{s}^{g}(A):=\left\{z \in C H^{g}(A)_{\mathbb{Q}} \mid k_{*}(z)=k^{s} z, \quad \forall k \in \mathbb{Z}\right\} .
$$

Then:

$$
C H^{g}(A)_{\mathbb{Q}}=C H_{0}^{g}(A) \oplus C H_{1}^{g}(A) \oplus \cdots \oplus C H_{g}^{g}(A) .
$$

Moreover $C H_{0}^{g}(A)=\mathbb{Q}\left\{0_{A}\right\}$ and $I=\bigoplus_{s \geq 1} C H_{S}^{g}(A)$ is the ideal, with respect to the Pontryagin product, of the zero-cycles of degree 0 . It is known that $I^{* r}=$ $\bigoplus_{s \geq r} C H_{s}^{g}(A)$ and that $I^{* 2}$ is the kernel of the albanese map tensored with $\mathbb{Q}$ :

$$
I \longrightarrow A_{\otimes Q}
$$

sending a zero cycle $\sum n_{i}\left\{a_{i}\right\}$ to the sum $\sum n_{i} a_{i}$ in $A$. Another useful property is that $C H_{s}^{g}(A) * C H_{t}^{g}(A)=C H_{s+t}^{g}(A)$.

We point out that the filtration $V_{1}(A) \subset V_{2}(A) \subset \cdots \subset A$ is, in some sense, induced by the filtration $\cdots \subset I^{* 2} \subset I \subset C H^{g}(A)_{\mathbb{Q}}$. Indeed, given a point $x \in A$ we use the notation:

$$
\{x\}=\left\{0_{A}\right\}+x_{1}+\cdots+x_{g}, \quad x_{i} \in C H_{i}^{g}(A) .
$$

Then we have:

Proposition 2.6 For all $x \in A$, $x$ belongs to $V_{k}(A)$ if and only if

$$
x_{k}=\cdots=x_{g}=0 \text {. }
$$

In particular $x \in V_{2}(A)$ if and only if $\{x\}-\left\{0_{A}\right\} \in C H_{1}^{g}(A)$.

Proof We apply to (1) the multiplication by $l$ in $A$ :

$$
\{l x\}=\left\{0_{A}\right\}+l x_{1}+\cdots+l^{g} x_{g} .
$$


Using this we have:

$$
\begin{aligned}
\left(\{x\}-\left\{0_{A}\right\}\right)^{* k}= & \sum_{i=0}^{k}(-1)^{i}\left(\begin{array}{l}
k \\
i
\end{array}\right)\{(k-i) x\} \\
= & \sum_{i=0}^{k}(-1)^{i}\left(\begin{array}{l}
k \\
i
\end{array}\right)\left(\left\{0_{A}\right\}+(k-i) x_{1}+\cdots+(k-i)^{g} x_{g}\right) \\
= & \sum_{i=0}^{k}(-1)^{i}\left(\begin{array}{l}
k \\
i
\end{array}\right)\left\{0_{A}\right\}+\sum_{i=0}^{k}(-1)^{i}\left(\begin{array}{l}
k \\
i
\end{array}\right)(k-i) x_{1}+\cdots \\
& +\sum_{i=0}^{k}(-1)^{i}\left(\begin{array}{l}
k \\
i
\end{array}\right)(k-i)^{g} x_{g} .
\end{aligned}
$$

Now we use the following formulas (see the proof of Lemma 3.3 in [12] or prove them by induction):

$$
\begin{aligned}
& \sum_{i=0}^{k}(-1)^{i}\left(\begin{array}{l}
k \\
i
\end{array}\right)(k-i)^{l}=0 \quad \text { if } l<k \\
& \sum_{i=0}^{k}(-1)^{i}\left(\begin{array}{l}
k \\
i
\end{array}\right)(k-i)^{k}=k !
\end{aligned}
$$

Therefore we obtain that:

$$
\left(\{x\}-\left\{0_{A}\right\}\right)^{* k}=k ! x_{k}+\cdots
$$

and similarly $\left(\{x\}-\left\{0_{A}\right\}\right)^{* l}=l ! x_{l}+\cdots$ for any $l \geq k$. Hence $x \in V_{k}(A)$ if and only if $x \in V_{l}(A)$ for all $l \geq k$ if and only if $x_{k}=\cdots=x_{g}=0$.

We have several consequences of this Proposition and of its proof:

Corollary 2.7 With the same notations we have that $x_{k}=\frac{1}{k !} x_{1}^{k}$. Hence $\{x\}=\exp \left(x_{1}\right)$. Moreover $x \in V_{k}$ if and only if $x_{k}=x_{1}^{k}=0$.

Proof We have seen along the proof of the Proposition that

$$
\left(\{x\}-\left\{0_{A}\right\}\right)^{* k}=k ! x_{k}+\cdots \text { higher degree terms. }
$$

Computing directly we get that

$$
\left(\{x\}-\left\{0_{A}\right\}\right)^{* k}=\left(x_{1}+\cdots+x_{k}\right)^{* k}=x_{1}^{k}+\cdots \text { higher degree terms. }
$$

By comparing both formulas we obtain the equality. 
Remark 2.8 Notice that our computations are more or less contained in [1]. Indeed define as in Sect. 4 of loc. cit. the map

$$
\gamma: A \longrightarrow I, \quad a \mapsto\{0\}-\{a\}+\frac{1}{2}(\{0\}-\{a\})^{* 2}+\frac{1}{3}(\{0\}-\{a\})^{* 3}+\cdots
$$

this is a morphims of groups. Then, with our notations, $\gamma(x)=-x_{1}$. In particular the image of $\gamma$ belongs to $C H_{1}^{g}(A)$.

Corollary 2.9 Let $a, b \in A$ be two points such that

$$
n\{a\}+m\{b\}=(n+m)\left\{0_{A}\right\}
$$

for some integers $1 \leq n, m$. Then $a, b \in V_{2}(A)$.

Proof Decomposing as before:

$$
\{a\}=\{0\}+a_{1}+\frac{1}{2} a_{1}^{2}+\cdots \quad\{b\}=\{0\}+b_{1}+\frac{1}{2} b_{1}^{2}+\cdots
$$

the equality of the statement implies that $n a_{1}+m b_{1}=0=n a_{1}^{2}+m b_{1}^{2}$. Then $b_{1}=-\frac{n}{m} a_{1}$ and thus $n a_{1}^{2}+\frac{n^{2}}{m^{2}} a_{1}^{2}=0$, so $a_{1}^{2}=b_{1}^{2}=0$ and $a, b \in V_{2}(A)$.

Corollary 2.10 Let $\varphi: A \longrightarrow B$ be an isogeny, then $\varphi^{-1}\left(V_{k}(B)\right) \subset V_{k}(A)$. In particular $\varphi\left(V_{k}(A)\right)=V_{k}(B)$ and $\varphi^{-1}\left(V_{k}(B)\right)=V_{k}(A)$.

Proof Since we work with Chow groups with rational coefficients it is clear that for an integer $n \neq 0$ the map $n_{*}: C H_{k}^{g}(A) \longrightarrow C H_{k}^{g}(A)$ is bijective. Let $\psi: B \longrightarrow A$ be an isogeny such that $\psi \circ \varphi=n$, we deduce that $\varphi_{*}: C H_{k}^{g}(A) \longrightarrow C H_{k}^{g}(B)$ is injective. Let $x \in \varphi^{-1}\left(V_{k}(B)\right)$ and set $\{x\}=\left\{0_{A}\right\}+x_{1}+\cdots+x_{g}$. By hypothesis

$$
\varphi(\{x\})=\left\{0_{B}\right\}+\varphi_{*}\left(x_{1}\right)+\cdots+\varphi_{*}\left(x_{g}\right) \in V_{k}(B) .
$$

Hence $\varphi_{*}\left(x_{k}\right)=0$ and $x_{k}=0$. Therefore $x \in V_{k}(A)$ and we are done.

Remark 2.11 This corollary also follows from the definition of $V_{k}$ and the fact that $\varphi_{*}$ is an isomorphism modulo torsion on Chow groups compatible with the Pontryagin product.

Another interesting consequence of this characterization is the following property:

Corollary 2.12 For any $0 \leq k, l \leq g$ we have that

$$
V_{k}(A)+V_{l}(A) \subset V_{k+l-1}(A) .
$$


Proof Let $x \in V_{k}(A), y \in V_{l}(A)$. Then $\{x\}=\left\{0_{A}\right\}+x_{1}+\cdots+x_{k-1}$ and $\{y\}=$ $\left\{0_{A}\right\}+y_{1}+\cdots+y_{l-1}$. Since $x_{i} * y_{j} \in C H_{i+j}^{g}(A)$ we obtain

$\{x+y\}=\{x\} *\{y\}=\left\{0_{A}\right\}+\left(x_{1}+y_{1}\right)+\left(x_{2}+x_{1} * y_{1}+y_{2}\right)+\cdots+x_{k-1} * y_{l-1}$.

Thus $x+y \in V_{k+l-1}(A)$.

As an application we have:

Corollary 2.13 Let $C$ be a hyperelliptic curve of genus $g$. Then

$$
\operatorname{dim} V_{k}(J C)=k-1
$$

for $1 \leq k \leq g$, that is, the maximal possible dimension is attained.

Proof Choosing a Weiestrass point to define the Abel-Jacobi map we can assume that the curve $C$ is contained in $V_{2}(J C)$, using inductively the previous Corollary, we have that

$$
C+\stackrel{(k-1)}{\cdots}+C=W_{k-1}^{0}(C) \subset V_{k}(J C)
$$

For instance, for the Jacobian of a genus 3 curve $C$ we have, in the hyperelliptic case, that $\operatorname{dim} V_{2}(J C)=1$ and $\operatorname{dim} V_{3}(J C)=2$. If instead $C$ is a generic quartic plane curve we have that $\operatorname{dim} V_{2}(J C)=0$ by Theorem 2.4 and $\operatorname{dim} V_{3}(J C) \geq 1$ by Remark 2.3, d. Denoting as in that remark $p$ a flex point and $q$ its residual point, we have also the following: for any $x \in C$ such that the tangent line to $C$ in $x$ goes through $q, x \in V_{2}(C)$ (we identify $C$ with the Abel-Jacobi image in $J C$ using $p$ ). Indeed: there exists a $y \in C$ with $2 x+y+q \sim 3 p+q$, hence in $J C$ there is a relation of the form $2\{x\}+\{y\}=3\{0\}$ and then Corollary 2.9 implies that $\{x\},\{y\} \in V_{2}(J C)$.

Assume now that $C$ is a quartic plane with a hyperflex, that is a point $p$ such that $\mathcal{O}_{C}(1) \cong \mathcal{O}_{C}(4 p)$. This condition defines a divisor in $\mathcal{M}_{3}$. Embed $C$ in its Jacobian using $p$ as base-point. Then for any bitangent $2 x+2 y$ we have $\{x\},\{y\} \in V_{2}(J C)$. Also, with the same argument, for a standard flex $q$ we have $\{q\} \in V_{2}(J C)$. Everything suggests that the points in " $C \cap V_{2}(J C)$ " could have some geometrial meaning. Notice that this leaves open the question whether the dimension of $V_{3}(J C)$ is 1 or 2 for a generic quartic plane curve $C$.

\section{A family of zero-cycles and the action on differential forms}

In this section we begin the proof of the main theorem. We proceed by contradiction, hence we assume that there exists an irreducible component $\mathcal{Y}$ of dimension $\geq 2 g$. By 2.4 we have $\operatorname{dim} V_{2}(A) \leq 1$, for any abelian variety $A$. Hence $V_{2}\left(A_{y}\right)$ contains curves for all $y \in \mathcal{Y}$ and, by hypothesis, at least one of these curves generates the abelian variety. By a standard argument (involving the properness and countability of relative 
Chow varieties and the existence of universal families of abelian varieties up to base change) we can assume the existence of the following diagram:

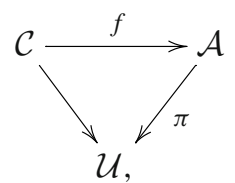

where the parameter space $\mathcal{U}$ comes equipped with a generically finite map $\Phi: \mathcal{U} \longrightarrow$ $\mathcal{Y}$ such that $\Phi(y)$ is the isomorphism class of $A_{y}$ for all $y \in \mathcal{U}$. Moreover $f_{y}: C_{y} \longrightarrow$ $A_{y}$ is the normalization map of an irreducible curve $f_{y}\left(C_{y}\right)$ contained in $V_{2}\left(A_{y}\right)$, and generating $A_{y}$, followed by the inclusion. We can also assume that $\mathcal{C} \longrightarrow \mathcal{U}$ has a section and then that $f$ induces a map of families of abelian varieties $F: \mathcal{J C} \longrightarrow \mathcal{A}$ over $\mathcal{U}$.

To start with we pull-back the families of curves and abelian varieties to $\mathcal{C}$ itself:

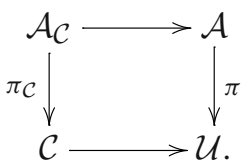

Now we define a family of zero-cycles in $\mathcal{A}_{\mathcal{C}}$ parametrized by $\mathcal{C}$. Let $s_{+}: \mathcal{C} \longrightarrow \mathcal{A}_{\mathcal{C}}$ be the section given by the maps $\left(i d_{\mathcal{C}}, f\right)$ :

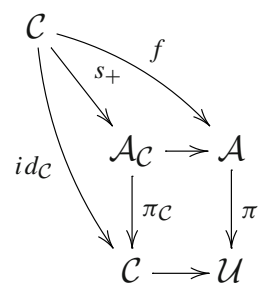

Put $\mathcal{Z}^{+}:=s_{+}(\mathcal{C})$. Analogously, by considering $-1_{\mathcal{A}} \circ f$, where $-1_{\mathcal{A}}$ is the relative -1 map on the family of abelian varieties we define a section $s_{-}: \mathcal{C} \longrightarrow \mathcal{A}_{\mathcal{C}}$ of $\pi_{\mathcal{C}}$ and a cycle $\mathcal{Z}^{-}:=s_{-}(\mathcal{C})$. Finally the zero section $0_{\mathcal{A}}$ induces a section $s_{0}$ and a cycle $\mathcal{Z}_{0}$. Set $\mathcal{Z}=\mathcal{Z}^{+}+\mathcal{Z}^{-}-2 \mathcal{Z}_{0}$, a cycle on $\mathcal{A}_{\mathcal{C}}$. In fact $\mathcal{Z}^{+}+\mathcal{Z}^{-}, 2 \mathcal{Z}_{0} \subset \operatorname{Sym}^{2} \mathcal{A}_{\mathcal{C}}$. Observe that $\mathcal{Z}_{t}, t \in \mathcal{C}$ is the 0 -cycle

$$
\mathcal{Z}_{t}=\{f(t)\}+\{-f(t)\}-2\left\{0_{A_{y}}\right\}
$$

on $A_{y}$, where $\pi(t)=y$. Since $f(t) \in f\left(C_{y}\right) \subset V_{2}\left(A_{y}\right)$ we have that $\mathcal{Z}_{t} \sim_{\text {rat }} 0$ in $A_{y}$ (see Proposition 2.5).

We are interested in an infinitesimal deformation of a curve $C_{y}$ for a general $y \in \mathcal{U}$. Thus, let us denote by $\Delta$ the spectrum of the ring of the dual numbers $\operatorname{Spec} \mathbb{C}[\varepsilon] /\left(\varepsilon^{2}\right)$. 
We consider a tangent vector $\xi \in T_{\mathcal{U}}(y)$ and we take a smooth quasi projective curve $B \subset \mathcal{U}$ passing through $y$ and with $\xi \in T_{B}(y)$. This induces the maps

$$
\alpha_{\xi}: \Delta \longrightarrow B \longrightarrow \mathcal{U}
$$

We pull-back to $B$ and to $\Delta$ the families of curves and abelian varieties and the cycle $\mathcal{Z}$, thus we have

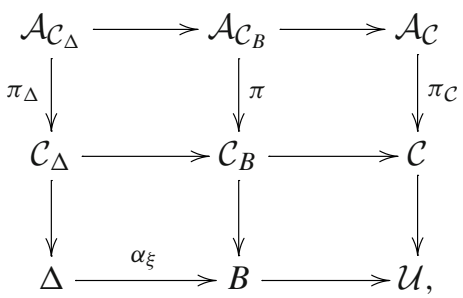

and $\mathcal{Z}_{\Delta}$ (resp. $\mathcal{Z}_{B}$ ) denotes the pull-back to $\mathcal{A}_{\mathcal{C}_{\Delta}}$ (resp. to $\mathcal{A}_{\mathcal{C}_{B}}$ ) of the cycle $\mathcal{Z}$.

The cycle $\mathcal{Z}_{B}$ determines a cohomological class $\left[\mathcal{Z}_{B}\right] \in H^{g}\left(\mathcal{A}_{\mathcal{C}_{B}}, \Omega_{\mathcal{A}_{\mathcal{C}_{B}}}^{g}\right)$ and its restriction a class $\left[\mathcal{Z}_{B}\right]_{t}$ in $H^{g}\left(A_{t}, \Omega_{\mathcal{A}_{\mathcal{C}_{B}} \mid A_{t}}\right)$, where $\pi(t)=y$.

The following is well-known by the experts and is a version of the classical results by Mumford and Roitman on zero-cycles (see [11, Lemma 2.2] and also [4]):

Proposition 3.1 If for any $t \in \mathcal{C}_{B}$, the restricted cycle $\mathcal{Z}_{t}$ is rationally equivalent to 0 , then there is a dense Zariski open set $\mathcal{V} \subset \mathcal{C}_{B}$ such that

$\left[\mathcal{Z}_{\mathcal{V}}\right]=0$ in $H^{g}\left(\mathcal{A}_{\mathcal{V}}, \Omega_{\mathcal{A}_{\mathcal{V}}}^{g}\right.$. In particularfor all $t \in \mathcal{V}$ we have $\left[\mathcal{Z}_{\mathcal{V}}\right]_{t}=\left[\mathcal{Z}_{B}\right]_{t}=0$ in $H^{g}\left(A_{t}, \Omega_{\mathcal{A}_{\mathcal{V}} \mid A_{t}}^{g}\right)$.

Now we look at the action of $\mathcal{Z}_{\Delta}$ in the space of differential forms on the infinitesimal family of abelian varieties. This works as follows.

Let $\mathcal{A}_{C_{y}}=A_{y} \times C_{y}$ be the restriction of $\mathcal{A}_{C_{\Delta}}$ over $C_{y}$. Consider $\Omega \in$ $H^{0}\left(\mathcal{A}_{C_{y}}, \Omega_{\mathcal{A}_{\mathcal{C}_{\Delta}} \mid \mathcal{A}_{C_{y}}}^{2}\right)$ and define:

$$
\mathcal{Z}_{\Delta}^{*}(\Omega)=\left(s_{+}\right)^{*}(\Omega)+\left(s_{-}\right)^{*}(\Omega)-2 s_{0}^{*}(\Omega)
$$

which belongs to $H^{0}\left(C_{y}, \Omega_{\mathcal{C}_{\Delta} \mid C_{y}}^{2}\right)$. Then we have by (3.1) that this action is trivial which gives the vanishing $\mathcal{Z}_{\Delta}^{*}(\Omega)=0$.

Consider also the family of abelian varieties on $\Delta$ :

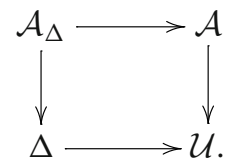


Notice that there is a natural map $\mathcal{A}_{\mathcal{C}_{\Delta}} \stackrel{h}{\rightarrow} \mathcal{A}_{\Delta}$ and that the composition:

$$
\mathcal{C}_{\Delta} \stackrel{s_{+}}{\rightarrow} \mathcal{A}_{\mathcal{C}_{\Delta}} \stackrel{h}{\rightarrow} \mathcal{A}_{\Delta}
$$

is simply the original family of curves $f_{\Delta}: \mathcal{C}_{\Delta} \longrightarrow \mathcal{A}_{\Delta}$ over $\Delta$. Therefore for any form $\Omega^{\prime} \in H^{0}\left(A_{y}, \Omega_{\mathcal{A}_{\Delta} \mid A_{y}}^{2}\right)$, denoting $\Omega=h^{*}\left(\Omega^{\prime}\right)$, we have that

$$
f_{\Delta}^{*}\left(\Omega^{\prime}\right)=s_{+}^{*}(\Omega)
$$

An almost identical computation can be done with $s_{-}$since $-1_{\mathcal{A}_{\mathcal{C}_{\Delta}}}$ acts trivially on the (2,0)-forms. Finally if $\Omega^{\prime} \in H^{0}\left(A_{y}, \Omega_{\mathcal{A}_{\Delta} \mid A_{y}}^{2}\right)^{0} \subset H^{0}\left(A_{y}, \Omega_{\mathcal{A}_{\Delta} \mid A_{y}}^{2}\right)$, is a form vanishing at the origin, then $s_{0}^{*}\left(h^{*}\left(\Omega^{\prime}\right)\right)=0$.

These considerations combined with (2) gives that for any form $\Omega^{\prime} \in H^{0}\left(A_{y}\right.$, $\left.\Omega_{\mathcal{A}_{\Delta} \mid A_{y}}^{2}\right)^{0}$ :

$$
\mathcal{Z}_{\Delta}^{*} h^{*}\left(\Omega^{\prime}\right)=2 f_{\Delta}^{*}\left(\Omega^{\prime}\right)
$$

Then, using the vanishing of $\mathcal{Z}^{*}(\Omega)$, this implies:

Proposition 3.2 With the above notations,

$$
f_{\Delta}^{*}: H^{0}\left(A_{y}, \Omega_{\mathcal{A}_{\Delta} \mid A_{y}}^{2}\right)^{0} \longrightarrow H^{0}\left(C_{y}, \Omega_{\mathcal{C}_{\Delta} \mid C_{y}}^{2}\right) \cong H^{0}\left(C_{y}, \omega_{C_{y}}\right)
$$

is the zero map.

We will see in the next section that, if the dimension of the family is $\geq 2 g$, then for a generic point of $\mathcal{U}$ and a convenient infinitesimal deformation there is a form in $H^{0}\left(A_{y}, \Omega_{\mathcal{A}_{\Delta} \mid A_{y}}^{2}\right)^{0}$ with non-trivial image in $H^{0}\left(C_{y}, \Omega_{\mathcal{C}_{\Delta} \mid C_{y}}^{2}\right)$. This contradicts the Proposition.

\section{The geometry of the adjoint form and end of the proof}

In this section we end the proof of the main Theorem. Assuming that the dimension of the family is $\geq 2 g$, we will find a contradiction with Proposition (3.2).

As at the beginning of section 3 we can assume the existence of the following diagram:

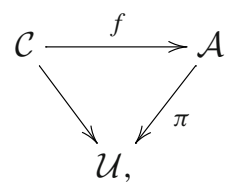

where the parameter space $\mathcal{U}$ comes equipped with a generically finite map $\Phi: \mathcal{U} \longrightarrow$ $\mathcal{Y}$. We fix a generic point $y$ in $\mathcal{U}$ and we denote by $\mathbb{T}$ the tangent space of $\mathcal{U}$ at $y$. 
Observe that $\mathbb{T} \hookrightarrow S y m^{2} H^{1,0}\left(A_{y}\right)^{*}$. Moreover the surjective map $F_{y}$ induces an inclusion of $W_{y}:=H^{1,0}\left(A_{y}\right)$ in $H^{0}\left(C_{y}, \omega_{C_{y}}\right)$. Let $D$ be the base locus of the linear system generated by $W_{y}$, therefore $W_{y} \subset H^{0}\left(C_{y}, \omega_{C_{y}}\left(-D_{y}\right)\right)$. Lemma 3.1 in [7] states that for a generic two dimensional subspace $E$ of $W_{y}$ the base locus of the pencil attached to $E$ is still $D_{y}$. As in the proof of Theorem 1.4 [7] we consider the map sending $\xi \in \mathbb{T}$, seen as a symmetric map $\cdot \xi: W_{y}=H^{1,0}\left(A_{y}\right) \longrightarrow H^{1,0}\left(A_{y}\right)^{*}$, to its restriction to $E$. Let $E_{0}$ be a complementary of $E$ in $W_{y}$. Then we have an element in $E^{*} \otimes E^{*}+E^{*} \otimes E_{0}^{*}$ which, by the symmetry, belongs to $S y m^{2} E^{*}+E^{*} \otimes E_{0}^{*}$. This last space has dimension $3+2(g-2)=2 g-1$. Since $\operatorname{dim} \mathcal{Y} \geq 2 g$ we get that the linear map

$$
\mathbb{T} \longrightarrow S y m^{2} E^{*}+E^{*} \otimes E_{0}^{*}
$$

sending $\xi$ to $\delta_{\xi \mid E}$ has non trivial Kernel. Therefore we conclude the following:

Lemma 4.1 For any 2-dimensional vector space $E \subset W_{y}$ there exists $\xi \in \mathbb{T}$ killing all the forms in $E$. Hence, if $\omega_{1}, \omega_{2}$ is a basis of $E$, then $\xi \cdot \omega_{1}=\xi \cdot \omega_{2}=0$.

We want to compute the adjunction form for a basis $\omega_{1}, \omega_{2}$ of $E$, as defined in [5]. Observe that $\xi$ can be seen as an infinitesimal deformation $\mathcal{A}_{\Delta}$ of $A_{y}$. We denote by $F_{\xi}$ the rank 2 vector bundle on $A_{y}$ attached to $\xi$ via the isomorphism $H^{1}\left(A_{y}, T_{A_{y}}\right) \cong$ $\operatorname{Ext}^{1}\left(\Omega_{A_{y}}^{1}, \mathcal{O}_{A_{y}}\right)$. With this notation the sheaf $F_{\xi}$ can be identified with $\Omega_{\mathcal{A}_{\Delta} \mid A_{y}}^{1}$. By definition there is a short exact sequence of sheaves:

$$
0 \longrightarrow \mathcal{O}_{A_{y}} \longrightarrow \Omega_{\mathcal{A}_{\Delta} \mid A_{y}}^{1} \longrightarrow \Omega_{A_{y}}^{1} \longrightarrow 0
$$

The connection map $H^{0}\left(A_{y}, \Omega_{A_{y}}^{1}\right) \longrightarrow H^{1}\left(A_{y}, \mathcal{O}_{A_{y}}\right)$ is the cup-product with $\xi \in$ $H^{1}\left(A_{y}, T_{A_{y}}\right)$. Then the forms $\omega_{1}, \omega_{2}$ lift to sections $s_{1}, s_{2} \in H^{0}\left(A_{y}, \Omega_{\mathcal{A}_{\Delta} \mid A_{y}}\right)$. These sections are not unique, but they are by imposing them to be 0 on the 0 -section of $\mathcal{A}_{\Delta} \longrightarrow \Delta$.

Then the adjoint form of $\omega_{1}, \omega_{2}$ with respect to $\xi$ is defined as the restriction of

$$
s_{1} \wedge s_{2} \in H^{0}\left(A_{y}, \Omega_{\mathcal{A}_{\Delta} \mid A_{y}}^{2}\right)^{0}
$$

to $C_{y}$. This is a section of $H^{0}\left(C_{y}, \Omega_{\mathcal{C}_{\Delta} \mid C_{y}}^{2}\right) \cong H^{0}\left(C_{y}, \omega_{C_{y}}\right)$.

Proposition 4.2 If the adjoint form vanishes then $\xi$ belongs to the kernel of $d \Phi$ : $\mathbb{T} \longrightarrow T_{\mathcal{A}_{g}}\left(A_{y}\right)=\operatorname{Sym}^{2} H^{1,0}\left(A_{y}\right)^{*}$.

Proof According to Theorem 1.1.8 in [5], the adjoint form vanishes if and only if the image of $\xi$ in

$$
H^{1}\left(C_{y}, T_{C_{y}}(D)\right) \cong \operatorname{Ext}^{1}\left(\omega_{C_{y}}(-D), \mathcal{O}_{C_{y}}\right)
$$


is zero. This says that the corresponding extension is trivial, so the short exact sequence in the first row of the next diagram splits (i.e. $i^{*} \Omega_{\mathcal{C}_{\Delta} \mid C_{y}}^{1}=\mathcal{O}_{C_{y}} \oplus \omega_{C_{y}}(-D)$ ):

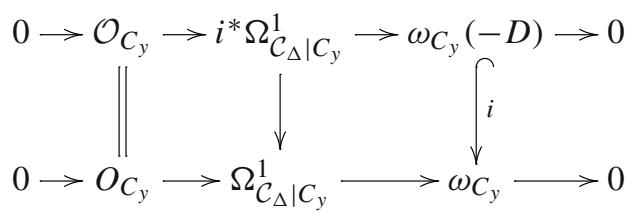

which implies that the connecting homomorphism in the associated long exact sequence of cohomology $H^{0}\left(C_{y}, \omega_{C_{y}}(-D)\right) \longrightarrow H^{1}\left(C_{y}, \mathcal{O}_{C_{y}}\right)$ is trivial. Therefore $\xi \cdot H^{0}\left(C_{y}, \omega_{C_{y}}(-D)\right)=0$ and in particular $\xi \cdot W_{y}=0$. Hence $\xi$ is in the kernel of $d \Phi_{y}$.

End of the proof of 1.1: Since $d \Phi$ is injective in a generic point, we are reduced to prove the vanishing of the adjoint form to reach a contradiction. Our aim is to use the vanishing obtained in Corollary (3.2).

We fix a generic point $y \in \mathcal{U}$ and consider $(\xi, E)$ as in Lemma (4.1). As in the Sect. 3, set $\Delta:=\operatorname{Spec} \mathbb{C}[\varepsilon] /\left(\varepsilon^{2}\right)$ and let $\alpha_{\xi} \longrightarrow \mathcal{U}$ be the map attached to $\xi$. From now on we restrict our family over $\mathcal{U}$ to a family over $\Delta$. Moreover we denote by $\mathcal{C}_{\Delta}$ the pull-back of $\mathcal{C}$ to $\Delta$, hence we have an infinitesimal deformation of $C_{y}$ :

$$
\mathcal{C}_{\Delta} \longrightarrow \Delta
$$

Again we pull-back to $\Delta$ the family of abelian varieties and the family of curves we get the diagram:

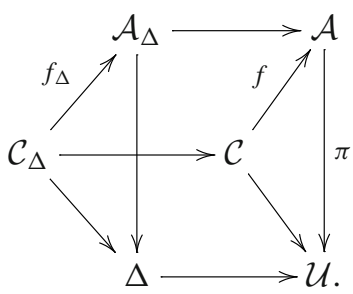

Notice that $E$ is generated by two linearly independent forms $\omega_{1}, \omega_{2} \in$ $H^{0}\left(A_{y}, \Omega_{A_{y}}^{1}\right) \subset H^{0}\left(C_{y}, \omega_{C_{y}}\right)$, we still denote by $s_{1}, s_{2} \in H^{0}\left(A_{y}, \Omega_{\mathcal{A}_{\Delta} \mid A_{y}}\right)$ the lifting of both sections. Then by the Proposition (3.2) the restriction to $C_{\Delta}$ of the form $s_{1} \wedge s_{2}$ is zero. Hence the adjoint form is zero and thus the Theorem is proved.

Remark 4.3 Since the Voisin set $V_{2}$ can be seen as the preimage of the rational orbit of the image of the origin in the corresponding Kummer variety our Theorem gives a bound on the dimension of the locus of Kummer varieties where these orbits are positive dimensional and "non-degenerate" (that is, the preimage in the abelian variety generates the abelian variety itself). 
Acknowledgements We warmly thank Olivier Martin for his careful reading of the paper, and the referee for valuable suggestions that have simplified and clarified the paper.

Open Access This article is licensed under a Creative Commons Attribution 4.0 International License, which permits use, sharing, adaptation, distribution and reproduction in any medium or format, as long as you give appropriate credit to the original author(s) and the source, provide a link to the Creative Commons licence, and indicate if changes were made. The images or other third party material in this article are included in the article's Creative Commons licence, unless indicated otherwise in a credit line to the material. If material is not included in the article's Creative Commons licence and your intended use is not permitted by statutory regulation or exceeds the permitted use, you will need to obtain permission directly from the copyright holder. To view a copy of this licence, visit http://creativecommons.org/licenses/by/4.0/.

\section{References}

1. Beauville, A.: Quelques remarques sur la transformation de Fourier dans l'anneau de Chow d'une variété abélienne, Algebraic geometry (Tokyo/Kyoto, 1982), 238-260, Lecture Notes in Mathematics, 1016, Springer, Berlin (1983)

2. Beauville, A.: Sur l'anneau de Chow d'une variété abélienne. Math. Ann. 273, 647-651 (1986)

3. Bloch, S.: Some elementary theorems about algebraic cycles on Abelian varieties. Invent. Math. 37, 215-228 (1976)

4. Bloch, S., Srinivas, V.: Remarks on correspondences and algebraic cycles. Am. J. Math. 105, 12351253 (1983)

5. Collino, A., Pirola, G.P.: The Griffiths infinitesimal invariant for a curve in its Jacobian. Duke Math. J. 78, 59-88 (1995)

6. Mumford, D.: Rational equivalence of zero-cycles on surfaces. J. Math. Kyoto Univ. 9, 195-204 (1969)

7. Naranjo, J.C., Pirola, G.P.: Hyperelliptic Jacobians and isogenies. Adv. Math. 335, 896-909 (2018)

8. Pirola, G.P.: Curves on generic Kummer varieties. Duke Math. J. 59, 73-80 (1989)

9. Roitman, A.A.: The torsion of the group of 0-cycles modulo rational equivalence. Ann. Math. (2) 111, 553-569 (1980)

10. Voisin, C.: Variations de structure de Hodge et zéro-cycles sur les surfaces générales. Math. Ann. 299, 77-103 (1994)

11. Voisin, C.: Infinitesimal invariants for cycles modulo algebraic equivalence and 1-cycles on Jacobians. Algebra Geom. 1, 140-165 (2014)

12. Voisin, C.: Chow rings and gonality of general abelian varieties. Ann. H. Lebesgue 1, 313-332 (2018)

Publisher's Note Springer Nature remains neutral with regard to jurisdictional claims in published maps and institutional affiliations. 